umgeht. Das Bedürfnis nach Zugehörigkeit/Bindung kann befriedigt werden, indem man am Leben der Kollegen und Mitarbeiter teilhat, sich mit dem Team identifiziert, sich loyal verhält, Interesse am Befinden der anderen zeigt, Verluste und Niederlagen gemeinsam betrauert oder Nähe und Vertrauen aufbaut.

\section{Nicht nur auf einen Lebensbereich fokussieren}

Wer zu viel in nur einen Lebensbereich (oft der Beruf) investiert, gerät in eine ungute Schräglage, in eine Lebensdysbalance, welche die psychophysische Gesundheit schädigen kann. Es fehlt der emotionale, kognitive und körperliche Ausgleich. In der Online-Befragung wurden die Teilnehmer aufgefordert anzugeben, in welchen Lebensbereich sie welchen Anteil ihrer $100 \%$ Lebensenergie investieren. Je mehr Energie ausschließlich in den Beruf fließt, umso größer ist - der Theorie nach - das Risiko eines berufsbedingten Burn-outs. Im Mittel wurden von den Teilnehmern $50 \%$ Lebensenergie in den Beruf investiert, 19\% in die eigene Person (eigenes Befinden, Interessen, Werte, Gesundheit etc.), $26 \%$ in Familie/
Partnerschaft und $14 \%$ in den Bereich Freundschaften.

Den kritischen Wert hinsichtlich Lebensdysbalance (nachhaltig gestörte Work-Life-Balance) erreichten oder überschritten $27 \%$ der verheirateten/verpartnerten und 39\% der ledigen Befragten. Einen hohen Wert im Hinblick auf einen Burn-out-fördernden Arbeitsstil hatten im Mittel 37,5\% dieser Befragten.

\section{Was die Umfragedaten offenbaren}

Neben den Zusammenhängen zwischen Stress und Arbeitszeit, Verträglichkeit der Arbeit mit anderen Lebensbereichen und fehlender beruflicher Zufriedenheit zeigen die Umfrageergebnisse weitere relevante Beziehungen. Für das Ausmaß von beruflichem Stress und damit auch für das Risiko, an einem Burn-out zu erkranken, sind demnach drei weitere Faktoren bedeutsam: die Work-Life-Balance, eine Balance der Bedürfnisse und ein Burn-out-fördernder Arbeitsstil.

Dysbalancen (v.a. das Fehlen einer Work-Life-Balance) und ein maladaptiver Arbeitsstil waren bei den Teilnehmern, die bei sich selbst ein Burn-out-Risiko sehen, erheblich höher. Hier wäre also prä- ventiv und interventiv zu handeln, um die Gesundheit am Arbeitsplatz zu erhalten und die Arbeitsqualität und -freude zu bewahren. Einschränkend ist zu erwähnen, dass sich überproportional häufig Ärzte an der Studie beteiligt haben könnten, die sich mit dem Thema Stress beschäftigen oder in Bezug auf eine Burnout-Gefährdung betroffen fühlen.

Das verzerrt womöglich die Datenlage dort, wo es um die Höhe oder Häufigkeit von Beschwerden geht, aber nicht da, wo Zusammenhänge zwischen Stress und Risikofaktoren ermittelt werden. Somit sollten die vorliegenden Ergebnisse Anstoß zu Veränderungen sein, die seitens der Arbeitgeber und seitens der betroffenen Ärzte gemeinsam und angepasst an die individuellen Bedingungen der Person und Arbeitsstätte umzusetzen wären.

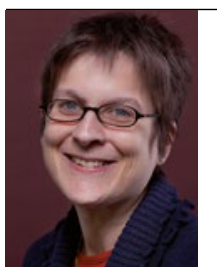

Dr. rer. nat. Dipl.-Psych. Katja Geuenich Akademie für Psychosomatik in der Arbeitswelt/ Röher Parkklinik Röherstraße 53 52249 Eschweiler

\title{
Forschungspreis für seltene Erkrankungen verliehen
}

Rund 4 Millionen Menschen leben in Deutschland mit einer seltenen Erkrankung. Aufgrund der geringen Patientenzahlen gibt es nur wenige Unternehmen der pharmazeutischen Industrie, die sich in Forschung und Entwicklung dieser Krankheitsbilder annehmen. Der "Eva Luise Köhler Forschungspreis" unterstützt darum jene wissenschaftlichen Projekte, die sich abseits großer Fallzahlen für Linderung und Heilung engagieren. Er wird von der "Eva Luise und Horst Köhler Stiftung" verliehen und ist mit $50.000 €$ dotiert.

In diesem Jahr geht der Forschungspreis an PD Dr. Sven Thoms und Prof. Dr. Ekkehard Wilichowski sowie das Forschungsteam der Arbeitsgruppe Muskeldystrophie der Klinik für Kinder- und Jugendmedizin der Universitätsmedizin Göttingen. Sie hatten die Jury mit ihrem Forschungsansatz zur Behandlung der Dysferlinopathie überzeugt. Ursache der Dysferlinopathie sind Mutationen im Dysferlin-Gen, die zu einem völligen Mangel oder zu Bildung eines abnormen Dysferlin-Proteins führen. Die Forscher gehen davon aus, dass das Reparaturprotein Dysferlin eine Schlüsselrolle bei Muskeldystrophien spielt. Der Körper benötigt dieses Eiweiß, um Verletzungen der Muskelzellmembran zu reparieren.

Die Arbeitsgruppe, insbesondere Dr. Julia Hofhuis und Kristina Bersch haben sich mit der Funktion von Dysferlin und den pathophysiologischen Mechanismen der Dysferlin-defizienten Muskeldystrophie in unterschiedlichen Modellsystemen beschäftigt. Darüber hinaus hat die Gruppe bereits eine Readthrough-Therapie bei Patienten mit Muskeldystrophie Typ Duchenne angewandt.

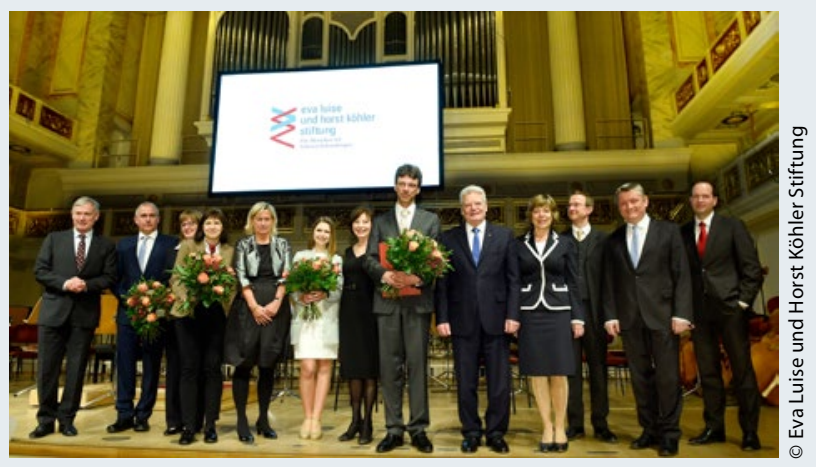

Die Preisträger des „Eva Luise Köhler Forschungspreises“ mit Bundespräsident Joachim Gauk und Daniela Schadt sowie dem ehemaligen Bundespräsidentenpaar Eva Luise und Horst Köhler 